\title{
Fractional Time Transportation Problem with Impurity Constraint
}

\author{
Madhuri Jain $^{1}$, Preetvanti Singh ${ }^{2}$, P. K. Saxena ${ }^{3}$ \\ ${ }^{1,2}$ (Faculty of Science, Dayalbagh Educational Institute, Dayalbagh Agra, India) \\ ${ }^{3}$ (Faculty of Engineering, Dayalbagh Educational Institute, Dayalbagh Agra, India)
}

\begin{abstract}
This paper presents theory and algorithm for solving a Fractional Time Transportation Problem with Impurity Constraint (FTTPI). The aim is to minimize the maximum of the total time that the various sources take to serve various destinations with certain amount of impurity presented in the commodities. The Fractional Time Transportation Problem with Impurity Constraint (FTTPI) is related to Lexicographic Fractional Time Transportation Problem with Impurity Constraint, which is solved by a lexicographic primal code. An algorithm is proposed to obtain a global optimal solution which is explained by solving a real life example.
\end{abstract}

Keywords: Time Transportation, Fractional Programming, Impurities, Lexicographic, Optimization.

\section{Introduction}

Transportation is one of the basic constituents of infrastructure of the country. It moves manpower and material among far-flung scattered locations of households and socio-economic activity centres. In this function, it improves mobility of inputs and their productivity which is vital for development of the economy. Different transportation models have been discussed by various researchers (Eboli and Mazzulla [6], Vincent and Jerram [12], Ziliaskopoulos and Waller [13], Barcelo et al. [2], etc. The Time Transportation Problem focuses attention on minimizing the time of transportation and its objective is to find a feasible distribution (of the supplies) which minimizes the maximum transportation time associated between a supply point and a demand point such that the distribution between the two points is positive. The time minimizing transportation problem is encountered in connection with transportation of perishable goods, with the delivery of emergency supplies, fire services, ambulance services or when military units are to be send from their bases to fronts. A bi-objective Cost-time Trade-off Three Axial Sums' Transportation Problem was shown to be equivalent to a singleobjective standard Three Axial Sums' problem by Bandopadhyaya [1]. Prakash et al. [9] considered a cost-time trade-off bulk transportation problem with the objectives to minimize the total cost and duration of bulk transportation without according priorities to them. Hochbaum and Woeginger [7] investigated a special case of the bottleneck transportation problem where the number $S$ of sources was bounded by a constant and not part of the input and demonstrated that for any fixed number $s \geq 1$, the bottleneck transportation problem with $S$ sources can be solved in linear-time. Poh et al. [8] described an approach to solve a real-world problem involving the transportation of multiple types of commodities from a number of sources to a number of destinations in discrete time periods, using a capacitated heterogeneous fleet of vehicles.

Transportation problems with fractional objective function are widely used as performance measures in many real life situations where an individual, or a group of community is faced with the problem of maintaining good ratios between some very important crucial parameters concerned with the transportation of commodities from certain sources to various destinations. In transportation problems, examples of fractional objectives (i.e. ratio objectives that have linear numerators and denominators) include optimization of total actual transportation cost/total standard transportation cost, total return/total investment, risk assets/capital, total tax/total public expenditure on commodity etc. Corban [1] extended the concept of multi-dimensional transportation problem with fractional linear objective function and derived the optimality conditions, for global optimum in terms of simplex multipliers. Chandra and Saxena [4] dealt with a technique for cost/completion date tradeoffs in a fractional transportation problem with penalty costs. An algorithm was also developed by reducing the fractional transportation problem with penalty costs to a transportation problem with objective function as a sum of a linear and linear fractional function. Tkacenko [11] proposed a generalized algorithm to solve a fractional multicriterial bottleneck transportation model. Basu and Acharya [3] studied bi-criterion quadratic fractional generalized solid transportation problem and developed an algorithm to obtain the time-cost trade-off pairs.

The transportation problems with restrictions in the commodity are very important from the practical point of view. In many real life situations, the commodity does vary in some characteristics according to its sources. The final commodity mixtures reaching the destinations may then be required to have known specifications. 
This paper presents Fractional Time Transportation Problem with Impurity Constraint where the commodity does vary in some characteristics according to its source and the final commodity mixture reaching various destinations may then be required to meet known specifications. An algorithm is also developed to solve such problems. The algorithm is supported by a real life example of Steel Transportation Problem of Steel Authority of India Limited (SAIL) were the problem is to determine a feasible transportation schedule which minimizes the Total Standard/Total Actual Transportation Time with Impurity Constraint.

\section{Mathematical Model}

Given a $\mathrm{M} \times \mathrm{N}$ Fractional Time Transportation Matrix, for transporting the goods from $\mathrm{i}^{\text {th }}(i=1,2, \ldots$, $M$ ) source point to $\mathrm{j}^{\text {th }}(j=1,2, \ldots, N)$ destination point, the problem is to find a feasible distribution (of the supplies) which minimizes the maximum fractional transportation time associated between a supply point and a demand point such that the distribution between the two points is positive while satisfying the extra requirement that the amount of nitrogen impurity present in steel is less than a certain critical level. The mathematical formulation of the Fractional Time Transportation Problem with Impurity Constraint (FTTPI) is

(FTTPI) $\min t=\max _{(i, j)}\left\{\frac{t_{i j}^{a}}{t_{i j}^{s}} \mid x_{i j}>0\right\}$

subject to

$$
\begin{array}{lc}
\sum_{j=1}^{N} x_{i j}=a_{i} & (i=1,2, \ldots M) \\
\sum_{i=1}^{M} x_{i j}=b_{j} & (j=1,2, \ldots N) \\
\sum_{i=1}^{M} f_{i j k} \cdot x_{i j} \leq q_{j k} & (j=1,2, \ldots N ; k=1,2, \ldots P) \\
x_{i j} \geq 0 & (i=1,2, \ldots M ; j=1,2, \ldots N)
\end{array}
$$

where $a_{i}$ is the quantity of the commodity available at the $i^{\text {th }}$ source and $b_{j}$ is the quantity of commodity required at the $j^{\text {th }}$ destination. One unit of the commodity contains $f_{i j k}$ units of $\mathrm{P}$ impurities $(k=1,2, \ldots P)$ when it is sent from the $i^{\text {th }}$ source to the $j^{\text {th }}$ destination. Customer $j$ cannot receive more than $q_{j k}$ units of impurity $k$ and $x_{i j}$ is the amount of the commodity transported from the $i^{\text {th }}$ source to the $j^{\text {th }}$ destination. $t_{i j}^{a} / t_{i j}^{s}$ is proportional contribution to the value of the fractional time objective function for shipping one unit of commodity from the $i^{t h}$ supply point to the $j^{\text {th }}$ demand point. Here $t_{i j}^{a}$ and $t_{i j}^{s}$ is independent of the commodity transported for $x_{i j}>0$.

Setting $M^{\prime}=\{1, \ldots M\}, N^{\prime}=\{1, \ldots N\}, J^{\prime}=\left\{(i, j) \mid i \in M^{\prime}, j \in N^{\prime}\right\}$ the FTTPI can be rewritten as

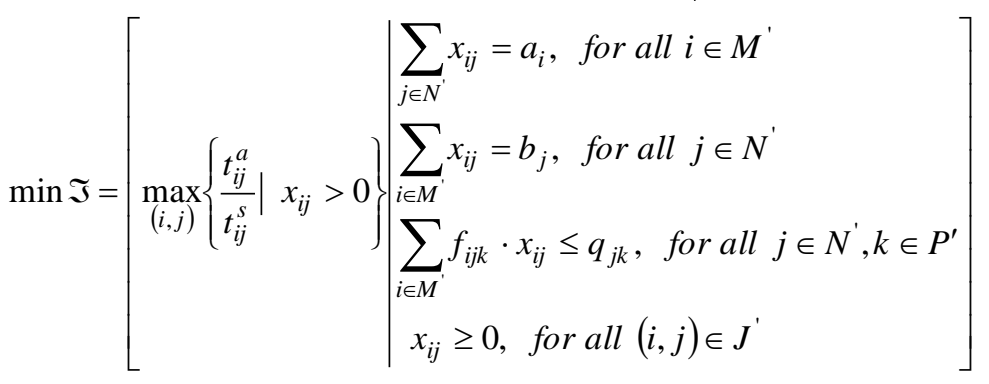

The assumptions here are if $a_{i} i \in M^{\prime}$ and $b_{j} j \in N^{\prime}$ are given non-negative numbers, then total supply quantity, $a_{i}$ is equal to the total demand quantity $b_{j}$. The denominator of the objective function of (6) is positive for all feasible solution and is always greater than or equal to the numerator. There are a total of $\mathrm{MN}+\mathrm{NP}$ variables including slacks and $\mathrm{NP}+\mathrm{M}+\mathrm{N}$ equations and a basic feasible solution consists of $\mathrm{NP}+\mathrm{M}+\mathrm{N}-1$ basic variables. 


\section{Lexicographic Fractional Time Transportation Problem with Impurity Constraint}

The FTTPI, where fractional time objective function is to be minimized, is formulated as a Lexicographic Fractional Time Transportation Problem with Impurity Constraint (LFTTPI):

$\operatorname{lexmin}\left[\begin{array}{l|l}\mathfrak{J}=\frac{\sum_{(i, j) \in J^{\prime}} \alpha_{i j} x_{i j}}{\sum_{(i, j) \in J^{\prime}} \beta_{i j} x_{i j}} \mid \begin{array}{l}\sum_{j \in N^{\prime}} x_{i j}=a_{i} \text { for all } i \in M^{\prime} \\ \sum_{i \in M^{\prime}} x_{i j}=b_{j} \text { for all } j \in N^{\prime} \leq q_{j k} \text { for all }(i, j) \in J^{\prime} \\ x_{i j} \geq 0 \text { for all }(i, j) \in J^{\prime}\end{array}\end{array}\right]$

with

$$
\alpha_{i j}:=\left[e_{c}\right] \quad(i, j) \in \xi_{c}^{a}
$$

$c=(1,2, \ldots g)$

$$
\text { and } \quad \beta_{i j}:=\left[e_{d}\right] \quad(i, j) \in \xi_{d}^{s} \text {, }
$$$$
d=(g+1, \ldots h)
$$

here $\alpha_{i j}, \beta_{i j} \in \mathfrak{R}^{h}, \mathfrak{R}^{h}$ be the set of real numbers.

Remark: Let $\mathfrak{R}$ denote the set of the real numbers and $\mathfrak{R}^{0}$ the set of the non-negative real numbers. With regard to lexicographic vector inequalities, the following convention will be applied: For $a, b \in \mathfrak{R}^{\hbar}$ the strict lexicographic inequality $a>b$ holds if $a_{\tilde{c}}>b_{\tilde{c}}$ and $a_{\tilde{d}}>b_{\tilde{d}}$ for $\tilde{c}=\min \left\{c \mid c=1,2, \ldots g, a_{c} \neq b_{c}\right\}$ and $\tilde{d}=\min \left\{d \mid d=g+1, g+2, \ldots h, a_{d} \neq b_{d}\right\}$ and the weak lexicographic inequality $\underset{\sim}{a \geq b}$ holds iff $\underset{\sim}{a>b}$ or $a=b$.

The additional impurity constraint (4) of FTTPI and also of LFTTPI can be written as $\sum_{i} f_{i j k} \cdot x_{i j}+x_{M+k, j}=q_{j k}$

$x_{M+k, j} \geq 0$

where $\mathrm{x}_{\mathrm{M}+\mathrm{k}, \mathrm{j}}$ are the slack variables to the impurity constraint.

\section{FRACTIONAL DUAL AND OPTIMALITY CONDITIONS}

Using the fractional dual of Swarup (1998), the Dual of Fractional Time Transportation Problem with Impurity constraint is derived as:

$\operatorname{lexmax} Q=\frac{\sum_{i \in M^{\prime}} a_{i} u_{i}^{1}+\sum_{j \in N^{\prime}} b_{j} v_{j}^{1}+\sum_{j \in N^{\prime}} \sum_{k \in P^{\prime}} w_{j k}^{1} q_{j k}}{\sum_{i \in M^{\prime}} a_{i} u_{i}^{2}+\sum_{j \in N^{\prime}} b_{j} v_{j}^{2}+\sum_{j \in N^{\prime}} \sum_{k \in P^{\prime}} w_{j k}^{2} q_{j k}}=\frac{V_{1}}{V_{2}}$

subject to

$\left[V_{2}\left\{\left(u_{i}^{1}+v_{j}^{1}+\sum_{k \in P^{\prime}} w_{j k}^{1} f_{i j k}\right)-\alpha_{i j}\right\}-V_{1}\left\{\left(u_{i}^{2}+v_{j}^{2}+\sum_{k \in P^{\prime}} w_{j k}^{2} f_{i j k}\right)-\beta_{i j}\right\}\right] \leq 0$

$\left[V_{2} \cdot w_{j k}^{1}-V_{1} \cdot w_{j k}^{2}\right] \leq 0$

$V_{2} \geq 0$

and

$$
u_{i}^{1}, u_{i}^{2}, v_{j}^{1}, v_{j}^{2}, w_{j k}^{1}, w_{j k}^{2} \text { are unrestricted in sign }
$$

where $u_{i}^{1}, u_{i}^{2}, v_{j}^{1}, v_{j}^{2}, w_{j k}^{1}, w_{j k}^{2}$ are vector-valued dual variables.

Now by the main duality theorem of fractional programming: 


$$
\begin{aligned}
& \frac{\sum_{(i, j) \in J^{\prime}} \alpha_{i j} x_{i j}}{\sum_{(i, j) \in J^{\prime}} \beta_{i j} x_{i j}}=\frac{\sum_{i \in M^{\prime}} a_{i} u_{i}^{1}+\sum_{j \in N^{\prime}} b_{j} v_{j}^{1}+\sum_{j \in N^{\prime}} \sum_{k \in P^{\prime}} w_{j k}^{1} q_{j k}}{\sum_{i \in M^{\prime}} a_{i} u_{i}^{2}+\sum_{j \in N^{\prime}} b_{j} v_{j}^{2}+\sum_{j \in N^{\prime}} \sum_{k \in P^{\prime}} w_{j k}^{2} q_{j k}} \\
& \text { Given } \sum_{(i, j) \in J^{\prime}} \beta_{i j} x_{i j}\left[\sum_{i \in M^{\prime}} a_{i} u_{i}^{1}+\sum_{j \in N^{\prime}} b_{j} v_{j}^{1}+\sum_{j \in N^{\prime} k \in P^{\prime}} w_{j k}^{1} q_{j k}\right]=\sum_{(i, j) \in J^{\prime}} \alpha_{i j} x_{i j}\left[\sum_{i \in M^{\prime}} a_{i} u_{i}^{2}+\sum_{j \in N^{\prime}} b_{j} v_{j}^{2}+\sum_{j \in N^{\prime} k \in P^{\prime}} w_{j k}^{2} q_{j k}\right] \\
& \Rightarrow \quad \sum_{(i, j) \in J^{\prime}}\left[V_{2}\left\{\left(u_{i}^{1}+v_{j}^{1}+\sum_{k \in P^{\prime}} w_{j k}^{1} f_{i j k}\right)-\alpha_{i j}\right\}-V_{1}\left\{\left(u_{i}^{2}+v_{j}^{2}+\sum_{k \in P^{\prime}} w_{j k}^{2} f_{i j k}\right)-\beta_{i j}\right\}\right] x_{i j} \\
& +\sum_{(i, j) \in J^{\prime}}\left[V_{2} \cdot w_{j k}^{1}-V_{1} \cdot w_{j k}^{2}\right] x_{M+k, j}=0
\end{aligned}
$$

From the dual constraints each term in equation (19) is less than or equal to zero. Hence

$\left\lfloor V_{2} \tilde{\alpha}_{i j}-V_{1} \tilde{\beta}_{i j}\right\rfloor x_{i j}=0$

and

$\left[V_{2} \cdot w_{j k}^{1}-V_{1} \cdot w_{j k}^{2}\right] x_{M+k, j}=0$

i.e. for $x_{i j}>0$,

$\left\lfloor V_{2} \tilde{\alpha}_{i j}-V_{1} \tilde{\beta}_{i j}\right\rfloor=0$

for $\mathrm{x}_{\mathrm{M}+\mathrm{k}, \mathrm{j}}>0$,

$\left[V_{2} \cdot w_{j k}^{1}-V_{1} \cdot w_{j k}^{2}\right]=0$

Therefore the optimality criteria is:

For basic variables

$$
\begin{aligned}
& \Delta_{i j}=\left[V_{2} \tilde{\alpha}_{i j}-V_{1} \tilde{\beta}_{i j}\right]=0 \\
& \Delta_{M+k, j}=\left[V_{1} \cdot w_{j k}^{2}-V_{2} \cdot w_{j k}^{1}\right]=0
\end{aligned}
$$

For non-basic variables

$$
\begin{aligned}
& \Delta_{i j}=\left|V_{2} \tilde{\alpha}_{i j}-V_{1} \tilde{\beta}_{i j}\right|_{\sim} 0 \\
& \Delta_{M+k, j}=\left[V_{1} \cdot w_{j k}^{2}-V_{2} \cdot w_{j k}^{1}\right] \underset{\sim}{\geq 0} \\
& \text { Where } \quad \tilde{\alpha}_{i j}=\alpha_{i j}-\left(u_{i}^{1}+v_{j}^{1}+\sum_{k \in P^{\prime}} w_{j k}^{1} \cdot f_{i j k}\right) \\
& \tilde{\beta}_{i j}=\beta_{i j}-\left(u_{i}^{2}+v_{j}^{2}+\sum_{k \in P^{\prime}} w_{j k}^{2} \cdot f_{i j k}\right) \\
& V_{1}=\left[\sum_{i \in M^{\prime}} \tilde{u}_{i}^{1} a_{i}+\sum_{j \in N^{\prime}} \tilde{v}_{j}^{1} b_{j}+\sum_{j \in N^{\prime} k \in P^{\prime}} \tilde{w}_{j k}^{1} \cdot q_{j k}\right] \\
& V_{2}=\left[\sum_{i \in M^{\prime}} \tilde{u}_{i}^{2} a_{i}+\sum_{j \in N^{\prime}} \tilde{v}_{j}^{2} b_{j}+\sum_{j \in N^{\prime} k \in P^{\prime}} \tilde{w}_{j k}^{2} \cdot q_{j k}\right]
\end{aligned}
$$

\section{Lemma 4.1}

If $\stackrel{*}{X}=\left(\begin{array}{l}* \\ x_{i j}, x_{M+k, j}\end{array}\right)(i, j) \in J^{\prime} ; k \in P^{\prime}$, is any feasible solution to F-component fractional time objective function $\Im$ in (7) and F-component vector valued variables $u_{i}, u_{i}, v_{j}, v_{j}, w_{j k}, w_{j k}$ be any feasible solution to F-component fractional time objective function $\mathrm{Q}$ defined as 
$\operatorname{lexmax} Q=\frac{\sum_{i \in M^{\prime}} a_{i} u_{i}^{1}+\sum_{j \in N^{\prime}} b_{j} v_{j}^{1}+\sum_{j \in N^{\prime}} \sum_{k \in P^{\prime}} w_{j k}^{1} q_{j k}}{\sum_{i \in M^{\prime}} a_{i} u_{i}^{2}+\sum_{j \in N^{\prime}} b_{j} v_{j}^{2}+\sum_{j \in N^{\prime}} \sum_{k \in P^{\prime}} w_{j k}^{2} q_{j k}}=\frac{V_{1}}{V_{2}}$

subject to

$\left[V_{2}\left\{\left(u_{i}^{1}+v_{j}^{1}+\sum_{k \in P^{\prime}} w_{j k}^{1} f_{i j k}\right)-\alpha_{i j}\right\}-V_{1}\left\{\left(u_{i}^{2}+v_{j}^{2}+\sum_{k \in P^{\prime}} w_{j k}^{2} f_{i j k}\right)-\beta_{i j}\right\}\right] y_{i j} \geq 0$

$\left[V_{2} \cdot w_{j k}^{1}-V_{1} \cdot w_{j k}^{2}\right] y_{M+k, j} \geq 0 \quad$ for all values of $\mathrm{k}$

$V_{2} \geq 0$

and $\quad u_{i}^{1}, u_{i}^{2}, v_{j}^{1}, v_{j}^{2}, w_{j k}^{1}, w_{j k}^{2}$ are unrestricted in sign

where $\left\{Y=\left(y_{i j} ; y_{M+k, j}\right) \in R_{0} \mid R_{0}\right.$ : set of non-negative numbers $\}$

$u_{i}^{1}, u_{i}^{2}, v_{j}^{1}, v_{j}^{2}, w_{j k}^{1}, w_{j k}^{2}$ are F-component vector-valued dual variables, and $\mathrm{V}_{1}$ and $\mathrm{V}_{2}$ are such that their corresponding F-components are not simultaneously zero. Let $\rho$ denote the set of all feasible solutions for Fcomponents fractional time objective function $\mathfrak{I}$ in (7) and ${ }^{*} \rho$ denote the set of all efficient solution for Fcomponent fractional time objective function $\mathrm{Q}$ in (32). Then

$$
\frac{\sum_{(i, j) \in J^{\prime}} \alpha_{i j}{ }^{*} x_{i j}}{\sum_{(i, j) \in J^{\prime}} \beta_{i j} x_{i j}} \leq \frac{\sum_{i \in M^{\prime}} a_{i}{ }^{* 1} u_{i}+\sum_{j \in N^{\prime}} b_{j} v^{* 1} v_{j}+\sum_{j \in N^{\prime} k \in P^{\prime}} \sum_{j k}^{* 1} w_{j k} q_{j k}{ }^{* 2} a_{i} u_{i}+\sum_{j \in N^{\prime}} b_{j}{ }^{* 2} v_{j}+\sum_{j \in N^{\prime} k \in P^{\prime}} \sum^{* 2} w_{j k} q_{j k}}{\sum^{\prime}}
$$

does not hold.

Proof: Since $x_{i j} \geq 0$ and $x_{M+k, j} \geq 0$, therefore from (7) and (10)



For no $X \in \rho$

Replacing the values 


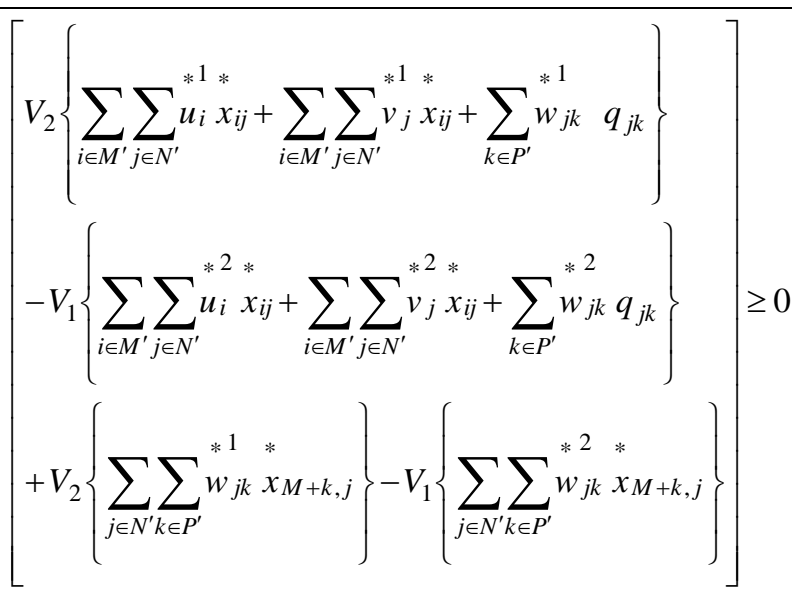

For no ${ }^{*} \in \rho$

And using (35)

$$
\frac{\sum_{(i, j) \in J^{\prime}} \alpha_{i j}{ }^{*} x_{i j}}{\sum_{(i, j) \in J^{\prime}} \beta_{i j}{ }^{*} x_{i j}} \leq \frac{\sum_{i \in M^{\prime}} a_{i} u_{i}^{* 1}+\sum_{j \in N^{\prime}} b_{j} v_{j}{ }^{* 1}+\sum_{j \in N^{\prime} k \in P^{\prime}} \sum_{j k}^{* 1} w_{j k} q_{j k}}{\sum_{i \in M^{\prime}} a_{i} u_{i}+\sum_{j \in N^{\prime}} b_{j} v_{j} v_{j}+\sum_{j \in N^{\prime} k \in P^{\prime}} \sum_{j k}^{* 2} q_{j k}}
$$

Hence the result.

\section{Theorem 4.2}

If $\stackrel{*}{X}=\left(\begin{array}{l}* \\ x_{i j}, x_{M+k, j}\end{array}\right)(i, j) \in J^{\prime} ; k \in P^{\prime}$ is any feasible solution to F-component fractional time objective function $\mathfrak{T}$ in (7). The solution $X$ is an efficient solution for F-component fractional time objective function $\mathfrak{J}$ of (7) iff $\exists$ a feasible solution $u_{i}, u_{i}\left(i \in M^{\prime}\right) ; v_{j}, v_{j}\left(j \in N^{\prime}\right) ; \quad w_{j k}^{* 1}, w_{j k}^{* 2}\left(j \in N^{\prime} ; k \in P^{\prime}\right)$ for F-component fractional time objective function $\mathfrak{I}$ of (7) such that.

$$
\frac{\sum_{(i, j) \in J^{\prime}} \alpha_{i j}{ }^{*} x_{i j}}{\sum_{(i, j) \in J^{\prime}} \beta_{i j}{ }^{*} x_{i j}}=\frac{\sum_{i \in M^{\prime}} a_{i} u_{i}^{* 1}+\sum_{j \in N^{\prime}} b_{j}{ }^{* 1} v_{j}+\sum_{j \in N^{\prime} k \in P^{\prime}} \sum_{* 1}^{* 1} w_{j k} q_{j k}}{\sum_{i \in M^{\prime}} a_{i} u_{i}+\sum_{j \in N^{\prime}} b_{j}{ }^{* 2} v_{j}+\sum_{j \in N^{\prime} k \in P^{\prime}} \sum^{* 2} w_{j k} q_{j k}}
$$

then the solution $u_{i}, u_{i}\left(i \in M^{\prime}\right) ; v_{j}, v_{j}\left(j \in N^{\prime}\right) ; w_{j k}, w_{j k}\left(j \in N^{\prime} ; k \in P^{\prime}\right)$ is itself an efficient solution for Fcomponent fractional time objective function $\mathrm{Q}$ in (32).

Proof: By Assumption

$$
\frac{\sum_{(i, j) \in J^{\prime}} \alpha_{i j}{ }^{*} x_{i j}}{\sum_{(i, j) \in J^{\prime}} \beta_{i j}{ }^{*} x_{i j}}=\frac{\sum_{i \in M^{\prime}} a_{i}{ }^{* 1} u_{i}+\sum_{j \in N^{\prime}} b_{j}{ }^{* 1} v_{j}+\sum_{j \in N^{\prime} k \in P^{\prime}} \sum_{j k}^{* 1} w_{j k} q_{j k}}{\sum_{i \in M^{\prime}} a_{i}{ }^{* 2} u_{i}+\sum_{j \in N^{\prime}} b_{j} v^{* 2}+\sum_{j \in N^{\prime} k \in P^{\prime}} \sum^{* 2} w_{j k} q_{j k}}
$$

Applying Lemma, for any feasible solution $\stackrel{*}{X}$ to F-component fractional time objective function $\mathfrak{I}$ in (7), 


$$
\begin{aligned}
& \frac{\sum_{(i, j) \in J^{\prime}} \alpha_{i j}{ }^{*} x_{i j}}{\sum_{(i, j) \in J^{\prime}} \beta_{i j}{ }^{*} x_{i j}}=\frac{\sum_{i \in M^{\prime}} a_{i} u_{i}^{* 1}+\sum_{j \in N^{\prime}} b_{j}{ }^{* 1} v_{j}+\sum_{j \in N^{\prime} k \in P^{\prime}} \sum^{* 1} w_{j k} q_{j k}}{\sum_{i \in M^{\prime}} a_{i}{ }^{2} u_{i}+\sum_{j \in N^{\prime}} b_{j}{ }^{* 2} v_{j}+\sum_{j \in N^{\prime} k \in P^{\prime}} \sum^{* 2} w_{j k} q_{j k}} \\
&=\frac{\sum_{(i, j) \in J^{\prime}} \alpha_{i j}{ }^{*}{ }^{x_{i j}}}{\sum_{(i, j) \in J^{\prime}} \beta_{i j}{ }^{*} x_{i j}} \quad \text { for no }{ }^{*} \in \in \rho
\end{aligned}
$$

Hence $\stackrel{*}{X} \in \stackrel{*}{\rho}$. Similar arguments hold for F-component fractional time objective function Q in (32).

\section{Theorem 4.3}

Let ${ }^{*}=\left(\begin{array}{l}* \\ x_{i j}, x_{M+k, j}\end{array}\right)(i, j) \in J^{\prime} ; k \in P^{\prime}$, be a feasible solution to F-component fractional time objective function $\Im$ in (7) if

$$
\begin{aligned}
& \sum_{\left(i, j \in J^{\prime}\right)}^{*} \Delta_{i j} y_{i j}<0, \quad y_{i j} \in R_{0} \text { for }(i, j) \in J^{\prime} \\
& \sum_{(i, j) \in J^{\prime}}^{*} \Delta_{M+k, j} y_{M+k, j}<0, \quad y_{M+k, j} \in R_{0} \text { for } j \in N^{\prime}, k \in P^{\prime}
\end{aligned}
$$

has no solution $Y=\left(y_{i j}, y_{M+k, j}\right)$, then $\stackrel{*}{X}$ is an efficient solution for F-component fractional time objective function $\Im$ in (7).

Proof: Let (40) and (41) have no solution i.e., the F-component vector valued multipliers in each $* 1 * 2 \quad * 1 * 2 \quad * 2$

system $u_{i}, u_{i}\left(i \in M^{\prime}\right) ; v_{j}, v_{j}\left(j \in N^{\prime}\right) ; w_{j k}, w_{j k}\left(j \in N^{\prime} ; k \in P^{\prime}\right)$; are feasible for F-component time objective function $\mathrm{Q}$ in (32). Since from the duality theorem

$$
\begin{aligned}
& \sum_{(i, j) \in J^{\prime}}^{*} \Delta_{i j} x_{i j}=0 \\
& \sum_{(i, j) \in J^{\prime}}^{*} \Delta_{M+k, j} x_{M+k, j}=0
\end{aligned}
$$

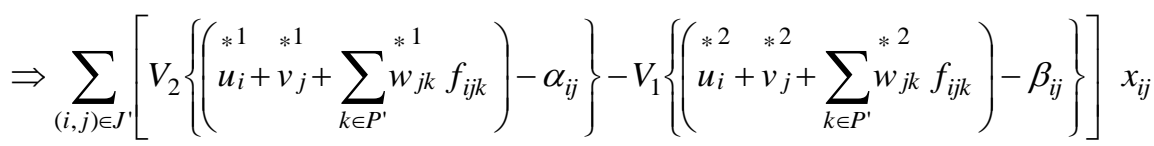

$$
\begin{aligned}
& \begin{array}{l}
+\sum_{(i, j) \in J^{\prime}}\left[V_{2} \cdot w_{j k}-V_{1} \cdot w_{j k}^{* 2}\right. \\
\Rightarrow \sum_{(i, j) \in J^{\prime}} \beta_{i j} x_{i j}\left[\sum_{(i, j) \in J^{\prime}}\left(\begin{array}{l}
* 1 \\
u_{i}+v_{j}+\sum_{k \in P^{\prime}}
\end{array} w_{j k} f_{i j k}\right) x_{i j}+\sum_{j \in N^{\prime} k \in P^{\prime}} \sum_{j k}^{* 1} x_{M+k, j}\right]-
\end{array} \\
& \sum_{(i, j)=J^{\prime}} \alpha_{i j} x_{i j}\left[\sum _ { ( i , j ) \in J ^ { \prime } } \left(\begin{array}{l}
* 2 \\
u_{i}+v_{j}
\end{array}\right.\right.
\end{aligned}
$$


holds for no $\mathrm{X} \in \rho$

$$
\begin{aligned}
& \Rightarrow \frac{\sum_{(i, j) \in J^{\prime}} \alpha_{i j} x_{i j}}{\sum \beta_{j} x_{i j}} \leq \frac{\sum_{i \in M^{\prime}} a_{i} u_{i} u_{i}+\sum_{j \in N^{\prime}} b_{j} v_{j}+\sum_{j \in N^{\prime} k \in P^{\prime}} \sum^{* 1} \mathrm{w}_{\mathrm{jk}} q_{j k}}{*^{2}{ }^{2}} \\
& \sum_{(i, j) \in J^{\prime}} \beta_{i j} x_{i j} \quad \sum_{i \in M^{\prime}} a_{i} u_{i}^{* 2}+\sum_{j \in N^{\prime}} b_{j} v_{j}^{* 2}+\sum_{j \in N^{\prime} k \in P^{\prime}} \sum_{\mathrm{jk}}^{* 2} q_{j k} \\
& \Rightarrow \frac{V_{1}}{V_{2}} \leq \frac{{ }^{*}}{V_{2}}
\end{aligned}
$$

Where last inequality follows from the fact that $V_{2}, V_{2}>0$. Hence ${ }_{X}^{*} \in \stackrel{*}{\rho}$.

\section{Altering a Feasible Basic Solution}

If a basic feasible solution is to be updated by introducing a non-basic variable and removing the basic one, then alterations can only be made to the basic variables. To determine the, incoming variable select the minimum

$$
\Delta_{i_{*} j_{*}}=\min \left\{\Delta_{i j} \mid \Delta_{i j}<0\right\}
$$

or

$$
\Delta_{M+k_{*}, j_{*}}=\min \left\{\Delta_{M+k, j} \mid \Delta_{M+k, j}<0\right\}
$$

By applying (42), the variables $x_{i_{k} j_{*}}$ or $x_{M+k_{*} j_{*}}$ becomes a basic variable of the new basic feasible solution, and an unknown quantity $\phi$ is to be added to this variable while $\phi \cdot \delta_{R S}$ or $\phi \cdot \delta_{M+Y, S}$ is added to all the basic variables $x_{R S}$ or $x_{M+Y, S}$. Then if the new solution satisfies the original constraints, the $\delta$ 's must satisfy the equations:

$$
\begin{array}{lc}
\sum_{R=1}^{M} \delta_{R S}=0 & (S=1,2, \ldots N) \\
\sum_{S=1}^{N} \delta_{R S}=0 & (R=1,2, \ldots M) \\
\sum_{Y=1}^{P} f_{i j k} \cdot \delta_{R S}+\delta_{M+Y, S}=0 & (S=1,2, \ldots N ; R=1,2 \ldots M)
\end{array}
$$

Here $\delta_{R S}=0$, if $x_{R S}$ is not in the basis and $\delta_{M+Y, S}=0$ if $x_{M+Y, S}$ is not in the basis. There are NP+M+N-1 independent equations in the set (43), (44) and (45) and NP+M+N unknown $\delta^{\prime} s$. It is therefore possible to solve this set of equations for the (M+N+NP-1) $\delta^{\prime} s$ associated with basic variables in terms of $\delta_{i_{k_{*}} j_{*}}$ or $\delta_{M+Y_{*} j_{*}}$ Furthermore, the values of the variables in the updated basic feasible solution are given by $x_{R S}+\delta_{R S} . \phi, x_{M+Y, S}+\delta_{M+Y, S} . \phi$. By choosing a suitable value of $\phi$ from

$$
\phi=\min _{\delta_{R S}<0}\left[-\frac{x_{R S}}{\delta_{R S}} ;-\frac{x_{M+Y, S}}{\delta_{M+Y, S}<0}\right]
$$

one of the variables is reduced to zero while the others remain positive and a new updated basic feasible solution is obtained.

The lexicographic sequence is as follows

$$
\begin{aligned}
& e_{1} e_{5}>e_{2} e_{5}>e_{1} e_{6}>e_{3} e_{5}>e_{2} e_{6}>e_{1} e_{7}>e_{4} e_{5}>e_{3} e_{6}>e_{2} e_{7}>e_{1} e_{8}>e_{4} e_{6}>e_{3} e_{7}>e_{2} e_{8}>e_{4} e_{7}>e_{3} e_{8}>e_{4} e_{8} \\
& \text { and } \frac{e_{1}}{e_{8}}>\frac{e_{2}}{e_{8}}>\frac{e_{1}}{e_{7}}>\frac{e_{3}}{e_{8}}>\frac{e_{2}}{e_{7}}>\frac{e_{1}}{e_{6}}>\frac{e_{4}}{e_{8}}>\frac{e_{3}}{e_{7}}>\frac{e_{2}}{e_{6}}>\frac{e_{1}}{e_{5}}>\frac{e_{4}}{e_{7}}>\frac{e_{3}}{e_{6}}>\frac{e_{2}}{e_{5}}>\frac{e_{4}}{e_{6}}>\frac{e_{3}}{e_{5}}>\frac{e_{4}}{e_{5}}
\end{aligned}
$$




\section{The FTTPI Algorithm}

The steps for solving FTTPI Algorithm, to enumerate optimal solution are as follows:

Step1: Determine the lower bound $t_{l}^{a}$ on $t^{a}$ and $t_{l}^{s}$ on $t^{s}$ to reduce the dimension of the vectors $\alpha_{i j}$ and $\beta_{i j}$ in eq. (7).

Step 2: Determine an initial feasible basic solution $X^{0}$ to LFTTPI by using method [13].

Step 3: From the resulting bottleneck time $t^{a}$ and $t^{s}$ of the initial feasible basic solution $X^{0}$, determine an upper bound $t_{u}^{a}$ and $t_{u}^{s}$.

Step 4: Partition the set $\xi^{a}=M \times N$ and $\xi^{s}=M \times N$ into subset $\xi_{d}^{a}$ and $\xi_{c}^{s}$ respectively $(c=1, \ldots g ; d=g+1, \ldots h)$. Each of the subset of $\xi_{c}^{a}$ consists of all $(i, j) \in \xi^{a}$ for which time of transportation $T=\left(t_{i j}^{a}\right)$ has the same numerical value. The subset $\xi_{1}^{a}$ contains all $(i, j) \in \xi^{a}$ with $t_{i j}^{a}$ being the highest value, subset $\xi_{2}^{a}$ contains all $(i, j) \in \xi^{a}$ with $t_{i j}^{a}$ being the next lower highest value, and so on. Finally subset $\xi_{g}^{a}$ contains all $(i, j) \in \xi^{a}$ with $t_{i j}^{a}$ being the lowest value. Now to each value of $x_{i j}$ with $(i, j) \in \xi^{a}(c=1,2, \ldots g)$, a $(g \times 1)$ unit vector $e_{c}$ is assigned for $(i, j) \in \xi_{c}^{a}(c=1,2, \ldots g)$. Same method is applied to determine the $\beta_{i j}$ for $\xi_{d}^{s}$ for $(i, j) \in \xi_{d}^{s}(d=g+1, g+2, \ldots h)$ and determine the vectors $\alpha_{i j}$ and $\beta_{i j}$ such that

$$
\alpha_{i j}=\left[e_{c}\right] \quad \text { and } \quad \beta_{i j}=\left[e_{d}\right]
$$

to obtain Fractional Time Matrix T.

Step 5: Designate the set of pairs of indices $(i, j)$ of the basic variable by I. Compute recursively the vectorvalued multipliers $u_{i}^{1}, u_{i}^{2}, v_{j}^{1}, v_{j}^{2}, w_{j k}^{1}, w_{j k}^{2}$ defined such that

$$
\begin{aligned}
& \alpha_{i j}-\left(u_{i}^{1}+v_{j}^{1}+\sum_{k \in P} w_{j k}^{1} \cdot f_{i j k}\right)=0 \\
& \beta_{i j}-\left(u_{i}^{2}+v_{j}^{2}+\sum_{k \in P} w_{j k}^{2} \cdot f_{i j k}\right)=0
\end{aligned}
$$

(for those $i, j$ for which $x_{i j}$ is in the basis)

$$
\begin{aligned}
& w_{j k}^{1}=0 \\
& w_{j k}^{2}=0
\end{aligned}
$$

(for those $j, k$ for which $x_{M+k, j}$ is in the basis)

Step 6: Let $U^{*}=\left(\tilde{u}_{i}^{1}, \tilde{u}_{i}^{2}, i \in M^{\prime} ; \tilde{v}_{j}^{1}, \tilde{v}_{j}^{2}, j \in N^{\prime} ; \tilde{w}_{j k}^{1}, \tilde{w}_{j k}^{2,} j \in N^{\prime} ; k \in P^{\prime}\right)$ be the solution of (47), (48), (49) and (50). Evaluate the relative criterion vectors

$$
\begin{aligned}
& \tilde{\Delta}_{i j}=\left[V_{2} \tilde{\alpha}_{i j}-\left.V_{1} \tilde{\beta}_{i j}\right|_{\sim} ^{\geq 0}\right. \\
& \tilde{\Delta}_{M+k, j}=\left[V_{1} \cdot w_{j k}^{2}-V_{2} \cdot w_{j k}^{1}\right] \underset{\sim}{\geq 0} \\
& \text { where } \quad \tilde{\alpha}_{i j}=\alpha_{i j}-\left(u_{i}^{1}+v_{j}^{1}+\sum_{k \in P} w_{j k}^{1} \cdot f_{i j k}\right) \\
& \tilde{\beta}_{i j}=\beta_{i j}-\left(u_{i}^{2}+v_{j}^{2}+\sum_{k \in P} w_{j k}^{2} \cdot f_{i j k}\right) \\
& V_{1}=\left[\sum_{i \in M^{\prime}} \tilde{u}_{i}^{1} a_{i}+\sum_{j \in N^{\prime}} \tilde{v}_{j}^{1} b_{j}+\sum_{j \in N^{\prime} k \in P^{\prime}} \tilde{w}_{j k}^{1} \cdot q_{j k}\right]
\end{aligned}
$$


$V_{2}=\left[\sum_{i \in M^{\prime}} \tilde{u}_{i}^{2} a_{i}+\sum_{j \in N^{\prime}} \tilde{v}_{j}^{2} b_{j}+\sum_{j \in N^{\prime} k \in P^{\prime}} \tilde{w}_{j k}^{2} \cdot q_{j k}\right]$

for all $(i, j) \in J^{\prime} / I$

Step 7: If all $\tilde{\Delta}_{i j}$ and $\tilde{\Delta}_{M+k, j}$ are lexicographically greater than or equal to the zero vector for all $(i, j) \in J^{\prime} / I$, the current feasible basic solution is optimal to LFTTPI and go to Step 10. Otherwise go to step 8 .

Step 8: Select

$$
\tilde{\Delta}_{i j_{*} j_{*}}=\min \left\{\tilde{A}_{i j} \mid \tilde{\Delta}_{i j}<0\right\}
$$

or

$\tilde{\Delta}_{M+k_{*} j_{*}}=\min \left\{\tilde{\Delta}_{M+k, j} \mid \tilde{\Delta}_{M+k, j}<0\right\}$

for all $(i, j) \in J^{\prime} / I$

By applying the selection rule (57) determine the variable $x_{i_{*} j_{*}}$ or $x_{M+k_{*} j_{*}}$ which is to enter. The variable $x_{i_{*} j_{*}}$ or $x_{M+k_{*} j_{*}}$ then becomes a basic variable of the new feasible basic solution.

Step 9: Change the current feasible basic solution to the new feasible basic solution using equations

$\sum_{R=1}^{M} \delta_{R S}=0$

$\sum_{S=1}^{N} \delta_{R S}=0$

$\sum_{Y=1}^{P} f_{i j k} \cdot \delta_{R S}+\delta_{M+Y, S}=0$

$\phi=\min _{\delta_{R S}<0}\left[-\frac{x_{R S}}{\delta_{R S}} ;-\frac{x_{M+Y, S}}{\delta_{M+Y, S}}\right]$

$\delta_{M+Y, S}<0$

go to Step5.

Step 10:If $\tilde{X}=\left(\tilde{x}_{i j}, \tilde{x}_{M+k, j}\right)$ is optimal transportation schedule for LFTTPI denoted by equation (6), then $\tilde{\mathfrak{J}}=\frac{\sum_{(i, j) \in J^{\prime}} \alpha_{i j} \tilde{x}_{i j}}{\sum_{(i, j) \in J^{\prime}} \beta_{i j} \tilde{x}_{i j}} \cdot \frac{\tilde{c}}{\tilde{d}}$ is the index of the first positive component of the optimal flow vector $\tilde{\mathfrak{J}}$. Also $\tilde{t}=\frac{\tilde{t}_{i j}^{a}}{\tilde{t}_{i j}{ }^{s}}$ with $(i, j) \in \xi_{\tilde{c}}^{a} / \xi_{\tilde{d}}^{s}$ is the optimal bottleneck fractional transportation time. The optimal transportation schedule $\tilde{X}=\left(\tilde{x}_{i j}\right) \frac{\widetilde{\mathfrak{J}}_{\tilde{c}}}{\widetilde{\mathfrak{J}}_{\tilde{d}}}=\sum_{(i, j) \in J}\left(x_{i j}\right) \quad$ (summing over all $(i, j) \in \xi_{\tilde{c}}^{a} / \xi_{\tilde{d}}^{s}$ ) represents the total distribution that requires the bottleneck time. The solution $\tilde{X}=\left(\tilde{x}_{i j}, \tilde{x}_{M+k, j}\right)$ is optimal for LFTTPI and, hence the optimal solution for FTTPI.

\section{Steel Transportation Problem of Steel Authority of India Limited (SAIL)}

The Algorithm, for determining the optimal solution to the problem, can be illustrated by considering the following example of SAIL. SAIL has different types of blast furnaces in each of the six work-centers (j), situated in Bhilai, Durgapur, Rourkela, Bumpur, Salem and Bhadravati in India. The work centre (j) is receiving a fixed quantity of steel (i) which has six different grades. The basic goal is to determine a feasible transportation schedule which minimizes the total standard/total actual arrival transportation time of steel, while satisfying the extra requirement that the amount of nitrogen impurity present in steel is less than a certain critical level. 
In Table 1, the total standard transportation time to total actual transportation time (in hours) required for transporting the steel from $i_{-}^{\text {th }}$ source to $j^{\text {th }}$ destination are displayed. Availabilities $a_{i}$ (in tonnage) and the nitrogen contents $p_{i}$ are listed in last column while requirements $b_{j}$ (in tonnage) and maximum nitrogen contents $\mathrm{L}_{\mathrm{j}}$ are shown in the last row. Let $\mathrm{x}_{\mathrm{ij}}$ be the Tonnage sent from $\mathrm{i}$ to $\mathrm{j}$ then it is required to

$\min t=\max _{(i, j)}\left\{\frac{t_{i j}^{a}}{t_{i j}^{s}} \mid x_{i j}>0\right\}$

subject to

$$
\begin{array}{lr}
\sum_{j=1}^{6} x_{i j}=a_{i} & (i=1,2, \cdots, 6) \\
\sum_{i=1}^{6} x_{i j}=b_{j} & (j=1,2, \cdots, 6) \\
\sum_{i=1}^{6} p_{i} \cdot x_{i j} \leq L_{j} b_{j} & \\
x_{i j} \geq 0 & (i=1,2, \cdots, 6 ; j=1,2, \cdots, 6)
\end{array}
$$

\begin{tabular}{|c|c|c|c|c|c|c|c|c|c|}
\hline & \multicolumn{6}{|c|}{ Work Centers $j$} & \multirow{2}{*}{$\begin{array}{c}\text { Tons } \\
\text { avail } \\
a_{i} \\
\end{array}$} & \multirow{2}{*}{$\begin{array}{c}\text { Nitrogen } \\
\text { Contents } \\
p_{i} \\
\end{array}$} \\
\hline & & 1 & 2 & 3 & 4 & 5 & 6 & & \\
\hline \multirow{12}{*}{ Steel i } & 1 & 5.58 & {$[5.50]$} & $\lceil 5.58$ & 5.17 & {$[5.83]$} & {$[5.58]$} & 6 & 0.4 \\
\hline & & $\overline{4.42}$ & $\overline{4.33}$ & $\overline{4.42}$ & $\overline{4.33}$ & $\overline{4.83}$ & $\overline{4.42}$ & & \\
\hline & 2 & 5.75 & 5.33 & 5.58 & 5.00 & 5.67 & 5.58 & 11 & 0.8 \\
\hline & & $\overline{4.42}$ & $\overline{4.33}$ & $\overline{4.42}$ & $\overline{4.42}$ & $\overline{4.42}$ & 4.33 & & \\
\hline & 3 & 5.25 & {$[5.58$} & 5.58 & 5.58 & 5.33 & {$[5.67]$} & 8 & 0.6 \\
\hline & & $\overline{4.33}$ & {$[\overline{4.42}$} & 4.33 & $\overline{4.33}$ & $\overline{4.42}$ & $\overline{4.00}$ & & \\
\hline & 4 & 5.67 & 5.00 & 5.17 & 5.58 & 5.67 & 5.58 & 5 & 0.4 \\
\hline & & $\overline{4.42}$ & $\overline{4.83}$ & $\overline{4.33}$ & $\overline{4.75}$ & $\lfloor\overline{4.42}$ & $\overline{4.42}$ & & \\
\hline & 5 & 5.58 & 5.67 & 5.58 & 5.33 & 5.50 & 5.58 & 1 & 0.6 \\
\hline & & 4.50 & 4.67 & 4.42 & 4.25 & $\overline{4.33}$ & $\overline{4.75}$ & & \\
\hline & 6 & 5.58 & 6.007 & 5.17 & 5.58 & 5.42 & 5.58 & 3 & 0.4 \\
\hline & & 4.42 & 4.75 & 4.58 & 4.08 & 4.33 & [4.42 & & \\
\hline \multicolumn{2}{|c|}{ Tons Reqd (bj) } & 7 & 10 & 9 & 4 & 1 & 3 & & \\
\hline \multicolumn{2}{|c|}{$\begin{array}{l}\text { Max Nitrogen } \\
(L j)\end{array}$} & 0.7 & 0.7 & 0.7 & 0.7 & 0.7 & 0.7 & & \\
\hline
\end{tabular}

Table 1 Data for FTTPI of Steel Shipping Problem

A lower bound for FTTPI is obtained by using method of [13]

$t_{l}^{s}=4.33$

Similarly

$$
t_{l}^{a}=5.58
$$

The initial feasible basic solution $X^{0}$ to FTTPI is 
$x_{11}=2, x_{12}=\frac{5}{2}, x_{14}=1, x_{16}=\frac{1}{2}, x_{22}=\frac{15}{2}, x_{23}=\frac{7}{2}, x_{33}=\frac{11}{2}, x_{36}=\frac{5}{2}, x_{41}=5$,

$x_{54}=1, x_{64}=2, . x_{65}=1, x_{71}=21, x_{73}=2, x_{74}=10, x_{75}=3, x_{76}=4$

With the resulting bottleneck transportation time, the upper bounds are

$t_{u}^{s}=4.42$

and $\quad t_{u}^{a}=5.67$

Hence $g=4$ and $h=4$ so $\xi^{a}$ and $\xi^{s}$ has four subsets:

$\begin{array}{ll}\xi_{1}^{a}:=\left\{(i, j) \in \xi^{a} \mid t_{i j}^{a}>5.67\right\} & \xi_{2}^{a}:=\left\{(i, j) \in \xi^{a} \mid t_{i j}^{a}=5.67\right\} \\ \xi_{3}^{a}:=\left\{(i, j) \in \xi^{a} \mid t_{i j}^{a}=5.58\right\} & \xi_{4}^{a}:=\left\{(i, j) \in \xi^{a} \mid t_{i j}^{a}<5.58\right\}\end{array}$

and

$$
\begin{array}{ll}
\xi_{5}^{s}:=\left\{(i, j) \in \xi^{s} \mid t_{i j}^{s}>4.42\right\} & \xi_{6}^{s}:=\left\{(i, j) \in \xi^{s} \mid t_{i j}^{s}=4.42\right\} \\
\xi_{7}^{s}:=\left\{(i, j) \in \xi^{s} \mid t_{i j}^{s}=4.33\right\} & \xi_{8}^{s}:=\left\{(i, j) \in \xi^{s} \mid t_{i j}^{s}<4.33\right\}
\end{array}
$$

The Fractional Time Matrix T of the following related Lexicographic Minimum Fractional Time Transportation Problem with Impurity Constraint (LFTTPI):

$\operatorname{lexmin}\left[\begin{array}{l|l}\mathfrak{I}=\frac{\sum_{i=1}^{6} \sum_{j=1}^{6} \alpha_{i j} x_{i j}}{\sum_{i=1}^{6} \sum_{j=1}^{6} \beta_{i j} x_{i j}} & \begin{array}{l}\sum_{j=1}^{6} x_{i j}=a_{i},(i=1,2, \cdots 6) \\ \sum_{i=1}^{6} x_{i j}=b_{j},(j=1,2, \cdots 6) \\ \sum_{i=1}^{6} p_{i} \cdot x_{i j} \leq L_{j} b_{j} \\ x_{i j} \geq 0\end{array}\end{array}\right]$

can be written as

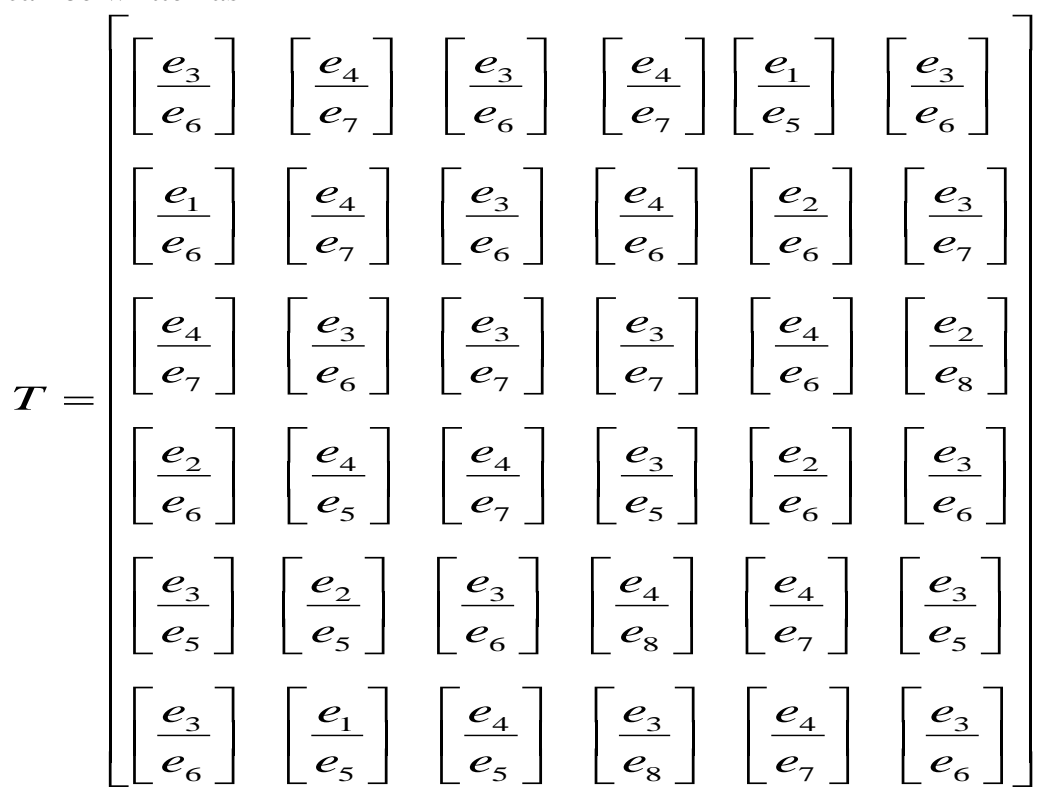

Using initial feasible basic solution $X^{0}$, the vector-valued multipliers $\tilde{u}_{i}^{1}, \tilde{u}_{i}^{2}, \tilde{v}_{j}^{1}, \tilde{v}_{j}^{2}$ and $w_{j k}^{1}, w_{j k}^{2}(i=1,2 \ldots .6 ; j=1,2 \ldots .6 ; k=1)$ are calculated as explained in Step 5: for cell $\mathrm{x}_{11}$ 


$$
\alpha_{11}=u_{1}^{1}+v_{1}^{1}+4 w_{11}^{1}
$$

and

$$
\beta_{11}=u_{1}^{2}+v_{1}^{2}+4 w_{11}^{2}
$$

giving $\quad v_{1}^{1}=e_{3} \quad$ and

$$
v_{1}^{2}=e_{6}
$$

Similarly other vector-valued multipliers are calculated. Then relative criterion vector $\tilde{\Delta}_{i j}$ is computed from equations (51) and (52):

$$
\begin{aligned}
& \Delta_{13}=\left[V_{2} \tilde{\alpha}_{13}-V_{1} \tilde{\beta}_{13}\right] \\
& \text { where } \quad \begin{aligned}
\tilde{\alpha}_{13} & =\alpha_{13}-\left(\tilde{u}_{1}^{1}+\tilde{v}_{3}^{1}+4 w_{31}^{1}\right) \\
& =e_{2}-e_{3}
\end{aligned}
\end{aligned}
$$

Similarly

$$
\tilde{\beta}_{13}=-e_{7}+e_{8}
$$

Also $\quad V_{1}=\frac{15}{2} e_{2}+\frac{27}{2} e_{3}+13 e_{4}$

$$
V_{2}=11 e_{6}+\frac{35}{2} e_{7}+\frac{11}{2} e_{8}
$$

Table 2 shows the transportation tableau with the initial feasible basic solution $X^{0}$. The amount $x_{i j}$ and $x_{M+k, j}$ are shown in the upper right corner of the cell. The marginal column contains the vector- valued multipliers $\frac{\tilde{u}_{i}^{1}}{\tilde{u}_{i}^{2}}$ and the marginal two rows contain $\frac{\tilde{v}_{j}^{1}}{\tilde{v}_{j}^{2}}$ and $\frac{\tilde{w}_{j k}^{1}}{\tilde{w}_{j k}^{2}}$. For all $(i, j) \in J^{\prime} / I$ the left corner contains $\tilde{\Delta}_{i j}$ and $\tilde{\Delta}_{M+k, j}$ if $\tilde{\Delta}_{i j}$ and $\tilde{\Delta}_{M+k, j}$ are lexicographically smaller than or equal to zero vectors. $L_{j}$ and $b_{j}$ are displayed in the top rows of the table, while $p_{i}$ and $a_{i}$ in the first and second columns respectively. The flow vector $\Im\left(\tilde{X}^{0}\right)=(0,5 / 2,0,2,0,0,1,11 / 2,5,0,12,6,0,0,0,0)^{T}$ indicates that bottleneck time $=1.334$ and bottleneck flow $=5 / 2$. As $\tilde{X}^{0}$ is not optimal, therefore applying the solution rule (57) of the Step 8 , the variable $x_{42}$ becomes the entering basic variable and so $\delta_{42}$ is added to this variable and $\delta_{R S}, \delta_{M+Y, S}$ is added to all the basic variables $x_{R S}, x_{M+Y, S}$. The $\delta$ 's satisfy the Eqs.(58)- (60) and can be solved in the following manner:

Here $\delta_{13}=0, \delta_{15}=0, \delta_{21}=0, \delta_{24}=0, \delta_{25}=0, \delta_{26}=0, \delta_{31}=0, \delta_{32}=0, \delta_{34}=0, \delta_{35}=0, \quad \delta_{43}=0, \delta_{44}=0 \quad \delta_{45}=0, \delta_{46}=0, \delta_{51}=0$, $\delta_{52}=0, \delta_{53}=0, \delta_{55}=0, \delta_{56}=0, \delta_{61}=0, \delta_{62}=0, \delta_{63}=0, \delta_{66}=0$ and $\delta_{72}=0$.

For $\mathrm{j}=1$,

$$
\begin{aligned}
\delta_{11}+\delta_{41} & =0 \\
4 \delta_{11}+4 \delta_{41}+\delta_{71} & =0
\end{aligned}
$$

For $\mathrm{j}=2$,

$$
\begin{gathered}
\delta_{12}+\delta_{22}+\delta_{42}=0 \\
4 \delta_{12}+8 \delta_{22}+4 \delta_{42}=0
\end{gathered}
$$

For $\mathrm{i}=2$,

$$
\delta_{22}+\delta_{23}=0
$$

For $\mathrm{j}=3$,

$$
\begin{aligned}
\delta_{23}+\delta_{33} & =0 \\
8 \delta_{23}+6 \delta_{33}+\delta_{73} & =0
\end{aligned}
$$

For $\mathrm{i}=3$,

$$
\delta_{33}+\delta_{36}=0
$$




\begin{tabular}{|c|c|c|c|c|c|c|c|c|}
\hline$p_{i}$ & $\mathrm{~L}_{\mathrm{i}}$ & 0.7 & 0.7 & 0.7 & 0.7 & 0.7 & 0.7 & $u_{-1}^{t}$ \\
\hline & $a b \rightarrow$ & 7 & 10 & 9 & 4 & 1 & 3 & $\overrightarrow{u^{2}}$ \\
\hline 0.4 & 6 & 2 & $5 / 2$ & $\begin{array}{l}\Delta_{13}= \\
(0,0,0,0 \\
11,0,0,-11 \\
25,0,0,-4 \\
-2,13,-19, \\
13)\end{array}$ & 1 & $\begin{array}{l}\Delta_{13}=(0,-15 / 2, \\
11,-272, \\
0,35 / 2,- \\
13,11, \\
15,11 / 2,-22, \\
892,-15 / 2, \\
-9,-8,-24)\end{array}$ & $1 / 2$ & $\frac{0}{0}$ \\
\hline 0.8 & 11 & $\begin{array}{l}\Delta_{11}=(0,0,11,0, \\
-11,352,0,0, \\
-25,112,0,- \\
27,2, \\
2,-13,272,13)\end{array}$ & $15 / 2$ & $7 / 2$ & $\begin{array}{l}\Delta_{24}=(0,0,0,0, \\
-372,0,0,-5 / 2, \\
-352,0,-13, \\
35 / 2,2,0,19,13)\end{array}$ & $\begin{array}{l}\Delta_{2}=(0,0,0,0, \\
15,2,0,0,17,2, \\
15,2,0,-35, \\
97,2,0,-22, \\
11,-11)\end{array}$ & $\begin{array}{l}\Delta_{2}=(0,0,0,0, \\
-72,0,0,492, \\
-652,0,13, \\
-192,2,-26 \\
19,13)\end{array}$ & $\frac{\varepsilon_{2}-\varepsilon_{3}}{-\varepsilon_{1}+\varepsilon_{3}}$ \\
\hline 0.6 & 8 & $\begin{array}{l}\Delta_{51}=(0,0,0,0, \\
-11,0,0,0, \\
-25,0,11,-27 / 2 \\
2,92,27 / 2,37 / 2)\end{array}$ & $\begin{array}{l}\Delta_{: 2}=(0,0,0,0, \\
-41 / 2,0,0,- \\
212, \\
52,0,-37,932, \\
1,2,15,1)\end{array}$ & $11 / 2$ & $\begin{array}{l}A_{24}=(0,0,0,0, \\
-372,0,0,172, \\
-352,0,-24,35, \\
2,-35 / 2,492, \\
15 / 2)\end{array}$ & $\begin{array}{l}\Delta_{33}=(0,0,0,0, \\
-26,0,0,-5, \\
-5 / 2,0,-37,62, \\
-11 / 2,17 / 2,11, \\
-11 / 2)\end{array}$ & $5 / 2$ & $\frac{\varepsilon_{2}-\varepsilon_{3}}{-\varepsilon_{6}+\varepsilon_{5}}$ \\
\hline 0.4 & 5 & 5 & $\begin{array}{l}\Delta_{4}=(0,- \\
152,2,0 \\
-272,-11,0,- \\
13, \\
11,-10,0,0,31 \\
112,13,112,0)\end{array}$ & $\begin{array}{l}\Delta_{43}=(0,0,0, \\
0,15 / 2,0,0, \\
5 / 2,0,0,24, \\
-35 / 2,-15 / 2, \\
35 / 2,-19,- \\
15 / 2)\end{array}$ & $\begin{array}{l}44=(0,-15 / 2, \\
0,-27 / 2,-11,0, \\
-13,22,-10,0, \\
-11,97 / 2,-11 / 2, \\
-92,11,-11 / 2)\end{array}$ & $\begin{array}{l}\Delta_{4}=(0,0,0,0, \\
152,0,0,17 / 2, \\
15,0,-35,62, \\
-15 / 2,-9,-5 / 2, \\
-24)\end{array}$ & $\begin{array}{l}\Delta_{4}=(0,0,0,0, \\
-11,0,0,11, \\
35 / 2,0,0,35 / 2, \\
112,0,112,0)\end{array}$ & $\frac{\varepsilon_{2}-\varepsilon_{3}}{0}$ \\
\hline 0.6 & 1 & $\begin{array}{l}\Delta_{2}=(0,-15 / 2,0, \\
-27 / 2,152,0 \\
-13,27 / 2,-15 / 2, \\
0,13,-27 / 2,15 / 2, \\
-13,27 / 2,13)\end{array}$ & $\begin{array}{l}\Delta_{22}=(0,- \\
15 / 2,0, \\
-27 / 2,332,0, \\
-13,-11 / 2,30, \\
0,-11,-2,12, \\
-11,4,1)\end{array}$ & $\begin{array}{l}\Delta_{y \mathrm{~s}}= \\
(0,0,0,0, \\
11,0,0,-11, \\
352,0,0,- \\
352, \\
112,0,- \\
112,0)\end{array}$ & 1 & $\begin{array}{l}\Delta y=(0,0,0,0, \\
0,0,0,11,0,0, \\
-11,352,0, \\
-35 / 2,11 / 2, \\
-11 / 2)\end{array}$ & $\begin{array}{l}\Delta_{96}=(0,- \\
15 / 2,0, \\
-27 / 2,15 / 2,0, \\
-13,27 / 2,- \\
15 / 2, \\
0,13,-27 / 2, \\
15 / 2,-13,27 / 2, \\
13)\end{array}$ & $\frac{0}{-\varepsilon_{7}+\varepsilon_{3}}$ \\
\hline 0.4 & 3 & $\begin{array}{l}\Delta_{51}=(0,0,0,0 \\
0,0,0,-11,-15 / 2 \\
0,11,-31,15 / 2 \\
92,8,37 / 2)\end{array}$ & $\begin{array}{l}\Delta_{52}=(0,-15 / 2, \\
11,-27 / 2,0, \\
352,-13,-11, \\
0,112,0,-35 / 2, \\
152,0,8,13)\end{array}$ & $\begin{array}{l}\Delta_{53}=(0,- \\
15 / 2, \\
0,- \\
27 / 2,372, \\
0,-13,-39 / 2, \\
352,0,35, \\
-105 / 2,112, \\
35,-33 / 2,11) \\
\end{array}$ & $\overline{2}$ & $\overline{1}$ & $\begin{array}{l}\Delta_{66}=(0,0,0,0, \\
0,0,0,-11,- \\
15,2, \\
0,11,-31,15 / 2, \\
92,8,37,2)\end{array}$ & $\frac{\varepsilon_{3}-\varepsilon_{4}}{-\varepsilon_{7}+\varepsilon_{3}}$ \\
\hline & & 21 & $\begin{array}{l}\Delta r=(0,0,0,0, \\
11: 4,0,0,-11 / 4, \\
25 / 4,0,0,-1, \\
-12,13,4, \\
-19,4,-13,4)\end{array}$ & 2 & 10 & 3 & 4 & \\
\hline$\frac{v_{j}^{1}}{v_{j}^{2}}$ & & $\frac{\varepsilon_{3}}{s_{6}}$ & $\frac{\varepsilon_{2}-\varepsilon_{3}+\varepsilon_{4}}{\varepsilon_{3}}$ & $\frac{-\varepsilon_{2}+2 \varepsilon_{3}}{\varepsilon_{6}+\varepsilon_{1}-\varepsilon_{3}}$ & $\frac{\varepsilon_{4}}{\varepsilon_{7}}$ & $\frac{-\varepsilon_{3}+2 \varepsilon_{4}}{2 \varepsilon_{7}-\varepsilon_{3}}$ & $\frac{\varepsilon_{3}}{\varepsilon_{6}}$ & \\
\hline
\end{tabular}

Table 2: Initial Solution

Similarly equations for $\mathrm{i}$ and $\mathrm{j}=4,5,6$ are written. Solving these equations:

$\delta_{11}=\delta_{42}, \delta_{12}=-\delta_{42}, \delta_{14}=0, \delta_{16}=0, \delta_{22}=0, \delta_{23}=0, \delta_{33}=0, \delta_{36}=0, \delta_{41}=-\delta_{42}, \delta_{54}=0$,

$\delta_{64}=0, \delta_{65}=0, \delta_{71}=0, \delta_{73}=0, \delta_{74}=0, \delta_{75}=0, \delta_{76}=0$

Using eq (61),

$\phi=\min (5 / 2,5)=5 / 2$ 
Using this value of $\delta_{42}$ the new feasible basic solution $\mathrm{X}^{1}$ is:

$$
X^{1}=\left[\begin{array}{lllllr}
9 / 2 & 0 & 0 & 1 & 0 & 1 / 2 \\
0 & 15 / 2 & 7 / 2 & 0 & 0 & 0 \\
0 & 0 & 11 / 2 & 0 & 0 & 5 / 2 \\
5 / 2 & 5 / 2 & 0 & 0 & 0 & 0 \\
0 & 0 & 0 & 1 & 0 & 0 \\
0 & 0 & 0 & 2 & 1 & 0 \\
21 & 0 & 2 & 10 & 3 & 4
\end{array}\right]
$$

Bottleneck flow $=5 / 2$, Time $=1.334$

All the values of $\tilde{\Delta}_{i j}$ and $\tilde{\Delta}_{M+k, j}$ are not lexicographically greater than or equal to zero, the solution is not optimal. Preceding further the optimal solution is obtained as $\mathrm{X}^{15}$ (Table 3).

$$
X^{2}=\left[\begin{array}{lllllr}
11 / 2 & 0 & 0 & 0 & 0 & 1 / 2 \\
0 & 15 / 2 & 7 / 2 & 0 & 0 & 0 \\
0 & 0 & 11 / 2 & 0 & 0 & 5 / 2 \\
3 / 2 & 5 / 2 & 0 & 1 & 0 & 0 \\
0 & 0 & 0 & 1 & 0 & 0 \\
0 & 0 & 0 & 2 & 1 & 0 \\
21 & 0 & 2 & 10 & 3 & 4
\end{array}\right]
$$

Bottleneck flow $=5 / 2$, Time $=1.334$

$$
X^{3}=\left[\begin{array}{cccccr}
6 & 0 & 0 & 0 & 0 & 0 \\
0 & 15 / 2 & 7 / 2 & 0 & 0 & 0 \\
0 & 0 & 11 / 2 & 0 & 0 & 5 / 2 \\
1 & 5 / 2 & 0 & 3 / 2 & 0 & 0 \\
0 & 0 & 0 & 1 / 2 & 0 & 1 / 2 \\
0 & 0 & 0 & 2 & 1 & 0 \\
21 & 0 & 2 & 11 & 3 & 3
\end{array}\right]
$$

Bottleneck flow $=5 / 2$, Time $=1.334$

$$
X^{4}=\left[\begin{array}{lccccc}
6 & 0 & 0 & 0 & 0 & 0 \\
0 & 15 / 2 & 7 / 2 & 0 & 0 & 0 \\
1 / 2 & 0 & 11 / 2 & 0 & 0 & 2 \\
1 / 2 & 5 / 2 & 0 & 2 & 0 & 0 \\
0 & 0 & 0 & 0 & 0 & 1 \\
0 & 0 & 0 & 2 & 1 & 0 \\
20 & 0 & 2 & 12 & 3 & 3
\end{array}\right]
$$

Bottleneck flow $=2$, Time $=1.334$

$$
X^{5}=\left[\begin{array}{cccccc}
6 & 0 & 0 & 0 & 0 & 0 \\
0 & 15 / 2 & 7 / 2 & 0 & 0 & 0 \\
1 & 0 & 11 / 2 & 0 & 0 & 3 / 2 \\
0 & 5 / 2 & 0 & 5 / 2 & 0 & 0 \\
0 & 0 & 0 & 0 & 0 & 1 \\
0 & 0 & 0 & 3 / 2 & 1 & 1 / 2 \\
19 & 0 & 2 & 12 & 3 & 4
\end{array}\right]
$$

Bottleneck flow $=3 / 2$, Time $=1.334$ 


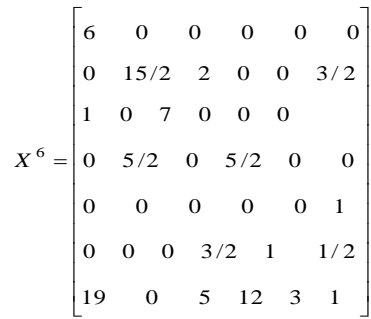

Bottleneck flow $=3 / 2$, Time $=1.313$

$$
X^{7}=\left[\begin{array}{cccccc}
6 & 0 & 0 & 0 & 0 & 0 \\
0 & 15 / 2 & 7 / 4 & 0 & 0 & 7 / 4 \\
1 & 0 & 7 & 0 & 0 & 0 \\
0 & 5 / 2 & 0 & 5 / 2 & 0 & 0 \\
0 & 0 & 0 & 0 & 0 & 1 \\
0 & 0 & 1 / 4 & 3 / 2 & 1 & 1 / 4 \\
19 & 0 & 6 & 12 & 3 & 0
\end{array}\right]
$$

Bottleneck flow $=3 / 2$, Time $=1.313$

$$
X^{8}=\left[\begin{array}{lccccc}
9 / 2 & 0 & 0 & 3 / 2 & 0 & 0 \\
0 & 15 / 2 & 7 / 4 & 0 & 0 & 7 / 4 \\
5 / 2 & 0 & 11 / 2 & 0 & 0 & 0 \\
0 & 5 / 2 & 0 & 5 / 2 & 0 & 0 \\
0 & 0 & 0 & 0 & 0 & 1 \\
0 & 0 & 7 / 4 & 0 & 1 & 1 / 4 \\
16 & 0 & 9 & 12 & 3 & 0
\end{array}\right]
$$

Bottleneck flow $=29 / 4$, Time $=1.289$

$$
X^{9}=\left[\begin{array}{lllllr}
17 / 4 & 0 & 0 & 3 / 2 & 0 & 1 / 4 \\
0 & 15 / 2 & 7 / 4 & 0 & 0 & 7 / 4 \\
11 / 4 & 0 & 21 / 4 & 0 & 0 & 0 \\
0 & 5 / 2 & 0 & 5 / 2 & 0 & 0 \\
0 & 0 & 0 & 0 & 0 & 1 \\
0 & 0 & 2 & 0 & 1 & 0 \\
31 / 2 & 0 & 19 / 2 & 12 & 3 & 0
\end{array}\right]
$$

Bottleneck flow $=7$, Time $=1.289$

$$
X^{10}=\left[\begin{array}{lclccc}
17 / 4 & 0 & 0 & 3 / 2 & 0 & 1 / 4 \\
0 & 15 / 2 & 7 / 4 & 0 & 0 & 7 / 4 \\
11 / 4 & 0 & 17 / 4 & 0 & 1 & 0 \\
0 & 5 / 2 & 0 & 5 / 2 & 0 & 0 \\
0 & 0 & 0 & 0 & 0 & 1 \\
0 & 0 & 3 & 0 & 0 & 0 \\
31 / 2 & 0 & 23 / 2 & 12 & 1 & 0
\end{array}\right]
$$

Bottleneck flow $=6$, Time $=1.289$

$$
X^{11}=\left[\begin{array}{lcccccc}
7 / 4 & 0 & 0 & 4 & 0 & 1 / 4 & \\
0 & 15 / 2 & 7 / 4 & 0 & 0 & 7 / 4 \\
21 / 4 & 0 & 17 / 4 & 0 & 1 & 0 \\
0 & 5 / 2 & 5 / 2 & 0 & 0 & 0 \\
0 & 0 & 0 & 0 & 0 & 1 \\
0 & 0 & 3 & 0 & 0 & 0 \\
21 / 2 & 0 & 33 / 2 & 12 & 1 & 0
\end{array}\right]
$$

Bottleneck flow $=3.5$, Time $=1.289$

$$
\text { www.iosrjournals.org }
$$




$$
X^{12}=\left[\begin{array}{ccccccc}
\in & 0 & 7 / 4 & 4 & 0 & 1 / 4 \\
0 & 15 / 2 & 7 / 4 & 0 & 0 & 7 / 4 \\
7 & 0 & 0 & 0 & 1 & 0 & \\
0 & 5 / 2 & 5 / 2 & 0 & 0 & 0 \\
0 & 0 & 0 & 0 & 0 & 1 \\
0 & 0 & 3 & 0 & 0 & 0 & \\
7 & 0 & 20 & 12 & 1 & 0
\end{array}\right]
$$

Bottleneck flow $=1.75$, Time $=1.289$

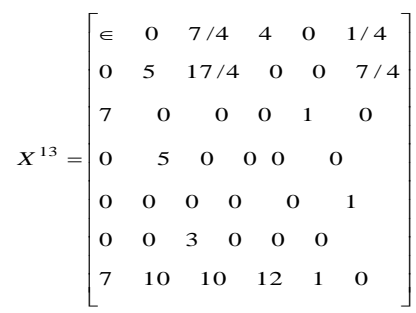

Bottleneck flow $=1.75$, Time $=1.289$

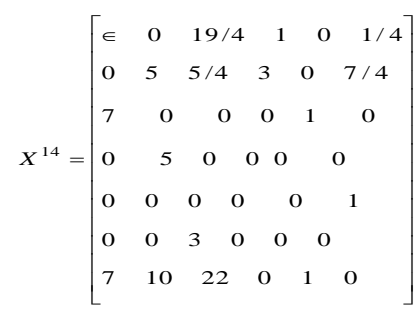

Bottleneck flow $=1.75$, Time $=1.289$

\begin{tabular}{|c|c|c|c|c|c|c|c|c|}
\hline$p_{i}$ & $\mathrm{~L}_{\mathrm{i}}$ & 0.7 & 0.7 & 0.7 & 0.7 & 0.7 & 0.7 & $u_{-}^{t}$ \\
\hline & $\downarrow_{\downarrow}^{a} b \rightarrow$ & 7 & 10 & 9 & 4 & 1 & 3 & $\overrightarrow{u^{2}}$ \\
\hline 0.4 & 6 & $\epsilon$ & $\begin{array}{l}\Delta_{12}=(0,0,0,0 \\
0,0,0,0 \\
0,0,0,0 \\
0,0,0,0)\end{array}$ & 3 & 1 & $\begin{array}{l}\Delta_{1 s}=(9,0,12, \\
-18,0,13, \\
-25,6,0,0,50, \\
-22,0,- \\
25,0,0)\end{array}$ & 2 & $\frac{0}{0}$ \\
\hline 0.8 & 11 & $\begin{array}{l}\Delta_{21}=(9,0,12,- \\
0,13,0,-12, \\
0,0,0,-13, \\
0,0,0,0)\end{array}$ & 5 & 3 & 3 & $\begin{array}{l}\Delta_{22}=(0,9,0,- \\
9, \\
12,0,0,-3, \\
13,0,25,-22, \\
0,-25,0,0)\end{array}$ & $\begin{array}{l}\Delta z=(0,0,0,0, \\
0,0,0,9, \\
0,0,25,-9, \\
0,-25,0,0)\end{array}$ & $\frac{0}{0}$ \\
\hline 0.6 & 8 & > & $\begin{array}{l}\Delta: 2=(0,0,0,18, \\
0,0,-18,6, \\
0,0,-74,44, \\
0,24,0,0)\end{array}$ & $\begin{array}{l}\Delta: 3=(0,0,0,9, \\
0,0,-9,12, \\
0,0,-12,13, \\
0,-13,0,0)\end{array}$ & $\begin{array}{l}\Delta_{34}=(0,0,0,18, \\
0,0,-18, \\
39 / 2,0,0, \\
-73 / 2,61 / 2, \\
0,-27 / 2,0,0)\end{array}$ & , & $\begin{array}{l}\Delta_{s 5}=(0,9,0,0, \\
12,0,-9,0, \\
13,0,-12,9, \\
0,12,-9,-25)\end{array}$ & $\frac{-e_{3}}{-e_{6}}$ \\
\hline 0.4 & 5 & $\begin{array}{l}\Delta_{4}=(0,9,0,0, \\
12,0,25,-12, \\
13,0,0,-22, \\
0,-25,0,0)\end{array}$ & 5 & $\begin{array}{l}\Delta_{43}=(0,0,0,0, \\
0,0,34,-3 \\
0,0,37,-31 \\
0,-37,0,0)\end{array}$ & $\begin{array}{l}\Delta_{44}=(0,0,0,9 \\
0,0,-9,12 \\
0,0,-12,13 \\
0,-13,0,0)\end{array}$ & $\begin{array}{l}\Delta_{4}=(0,9,0,0, \\
12,0,25,-3, \\
13,0,25,-31 \\
0,-50,0,0)\end{array}$ & $\begin{array}{l}\Delta_{45}=(0,0,0,9, \\
0,0,25,0 \\
0,0,0,-9, \\
0,-25,0,0)\end{array}$ & $\frac{0}{s_{s}}$ \\
\hline
\end{tabular}

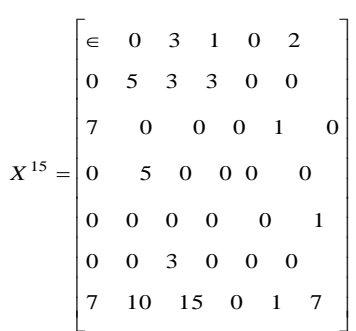

Bottleneck flow $=13$, Time $=1.270$ 
Fractional Time Transportation Problem With Impurity Constraint

\begin{tabular}{|c|c|c|c|c|c|c|c|c|}
\hline 0.6 & 1 & $\begin{array}{l}\Delta_{s 1}=(0,0,0,0, \\
0,0,0,0, \\
0,0,0,0, \\
0,0,0,0)\end{array}$ & $\begin{array}{l}\Delta_{92}=(0,9,0,0, \\
12,0,-9,-9, \\
13,0,-37,9, \\
0,12,0,0)\end{array}$ & $\begin{array}{l}\Delta_{s 3}=(0,0,0,9, \\
0,0,25,-9, \\
0,0,-25,0, \\
0,0,0,0)\end{array}$ & $\begin{array}{l}\Delta_{s 4}=(0,0,0,9, \\
0,0,25,-92, \\
0,0,-25 / 2,92, \\
0,25 / 2,-9,-25)\end{array}$ & $\begin{array}{l}\Delta_{y s}=(0,0,0,0, \\
0,0,34,-3, \\
0,0,37,-31 \\
0,-37,0,0)\end{array}$ & 1 & $\frac{0}{s_{9}-}$ \\
\hline \multirow[t]{2}{*}{0.4} & 3 & $\begin{array}{l}\Delta_{61}=(0,0,0,18, \\
0,0,16,3, \\
0,0,-37,13, \\
0,-13,0,0)\end{array}$ & $\begin{array}{l}\Delta_{52}=(9,0,12,9, \\
0,13,-18,3, \\
0,0,-49,22, \\
0,-1,0,0)\end{array}$ & 3 & $\begin{array}{l}\Delta_{84}=(0,0,0,27, \\
0,0,7,15, \\
0,0,-49,35, \\
0,-1,-9,-25)\end{array}$ & $\begin{array}{l}\Delta_{58}=(0,0,0,9, \\
0,0,25,9, \\
0,0,25,-18, \\
0,-50,0,0)\end{array}$ & $\begin{array}{l}\Delta_{56}=(0,0,0,18, \\
0,0,16,3, \\
0,0,-37,13, \\
0,-13,0,0)\end{array}$ & $\frac{-\varepsilon_{3}}{e_{3}}$ \\
\hline & & 7 & 10 & 15 & $\begin{array}{l}\Delta-4=(0,0,0,0, \\
0,0,0,94, \\
0,0,25 / 4,-9 / 4, \\
0,-25 / 4,0,0)\end{array}$ & 4 & 7 & \\
\hline$\frac{v_{j}^{1}}{v_{j}^{2}}$ & & $\frac{\varepsilon_{3}}{\varepsilon_{6}}$ & $\frac{e_{4}}{\varepsilon_{7}}$ & $\frac{\varepsilon_{3}}{\varepsilon_{6}}$ & $\frac{\varepsilon_{4}}{-\varepsilon_{6}+2 \varepsilon_{7}}$ & $\frac{\varepsilon_{3}}{2 \varepsilon_{6}-\varepsilon_{7}}$ & $\frac{\varepsilon_{3}}{\varepsilon_{6}}$ & \\
\hline$\frac{w_{j k}^{1}}{w_{j k}^{2}}$ & & 0 & 0 & 0 & $\frac{0}{\frac{e_{6}}{4}-\frac{e_{7}}{4}}$ & 0 & 0 & \\
\hline
\end{tabular}

Table 3: Optimal Solution

\section{Conclusion}

The developed FTTPI Algorithm solves fractional time transportation problem with impurity constraints and will help the Transportation System Decision Maker in determining the optimal transportation schedule with respect to the minimization of non-linear time function. The algorithm minimizes the vector of partial flows in a lexicographic sense on the feasible set. The optimal flow specifies the minimal bottleneck transportation time and the minimal flow which requires the optimal bottleneck transportation time. This paper also gives an interesting real life application of Fractional Time Dual having impurity constraints.

\section{References}

[1] L. Bandopadhyaya, Cost-time trade-off in three-axial sums transportation problem, Austral. Math. Soc. Ser. B, 35 (1994) $498-505$.

[2] J. Barcelo, E. Codina, J. Casas, J .L. Ferrer and D. Garcia, Microscopic traffic simulation: A tool for the design, analysis and evaluation of intelligent transport systems, Journal of Intelligent and Robotic Systems, 41 (2004) 173-203.

[3] M. Basu and D.P. Acharya, On quadratic fractional generalized solid bi-criterion transportation problem", J. Appl. Math. \& Computing, 10(1-2) (2002) 131-143.

[4] S. Chandra and P. K. Saxena, Fractional Transportation Problem with Penalty Costs and Impurities, Advances in Management Studies, 3(1), (1984) 57-65.

[5] A Corban, Programming with fractional linear objective function, Revue Roumainee de Mathematiques Pures et Appliquees, 18 (1973) 633-637.

[6] L. Eboli and G. Mazzulla, Service quality attributes affecting customer satisfaction for bus transit, Journal of Public Transportation, 10(3) (2007) 21-34.

[7] D. S. Hochbaum and G.J. Woeginger, A linear-time algorithm for the bottleneck transportation problem with a fixed number of sources", Operations Research Letters, 24(1-2) (1999) 25-28.

[8] K. L. Poh, K.W. Choo and C.G. Wong, A heuristic approach to the multi-period multi-commodity transportation problem, The Journal of the Operational Research Society, 56(6) (2005) 708-718.

[9] S. Prakash, P. Kumar, B.V.N.S. Prasad and A. Gupta, Pareto optimal solutions of a cost-time trade-off bulk transportation problem", European Journal of Operational Research, 188(1) (2008) 85-100.

[10] K. Swarup, Duality in fractional programming, Unternehmensforschung, 12 (1968) 106-112.

[11] A. I. Tkacenko, The generalized algorithm for Solving the fractional multi-objective transportation problem, ROMAI J. 2(1) (2006) 197-202.

[12] W. Vincent and L.C. Jerram, The potential for bus rapid transit to reduce transportation-related $\mathrm{CO}_{2}$ emissions, Journal of Public Transportation, BRT Special Edition, (2006) 219-237.

[13] A. K. Ziliaskopoulos and S.T. Waller, An Internet-based geographic information system that integrates data, models and users for transportation applications", Transportation Research Part C, 8, (2000) 427-444. 